\title{
VOLKER DEUBEL
}

Friedrich Schlegel: Kritische Ausgabe seiner Werke. Bd. 25: Höhepunkt und Zerfall der romantischen Schule (I799-1802). Mit Einleitung und Kommentar hg. von Hermann Patsch. Paderborn/München u. a.: Schöningh 2009. LXXXIV+738 S. € I 48,-. ISBN 978-3-506-77825-3.

Wenn es zutrifft, dass Kritische Editionen die Fundamente bilden, auf denen die Gebäude interpretierender Forschung errichtet werden ${ }^{1}$, so hat besonders die Romantikforschung dem Erscheinen des von Hermann Patsch herausgegebenen Briefbands der Kritischen Friedrich-Schlegel-Ausgabe viel zu verdanken. Denn der dokumentierte Zeitraum - es geht um die Jahre von Herbst 1799 bis Sommer I 802 - führt in einen ihrer Kernbereiche. Es handelt sich um die entscheidenden Jahre der Frühromantik, der Titel annonciert nicht weniger als »Höhepunkt « und $\gg$ Zerfall «, das entscheidende Kapitel in der Geschichte der Schule. Aber auch die Schlegelforschung erhält Auftrieb, wenn sie mit ihrer - immer noch nicht erledigten - zentralen Frage nach der Einheit des Gesamtwerks gerade auch auf die Schriften dieses Zeitraums blickt, Bruchstellen oder Verbindungsglieder zwischen Früh- und Spätwerk sucht oder sogar bereits Weichenstellungen für die Konversion zur katholischen Kirche zu erkennen glaubt.

So stellt sich die Frage, ob Erwartungen dieser Art durch den vorliegenden Band erfüllt werden können. Die editorische Ausgangslage ist eher problematisch zu nennen. Unter den 263 Briefen des Bandes befinden sich lediglich acht, die auf neue Funde zurückgehen. Das Gros war bereits in Buchform, teilweise in wissenschaftlichen Ausgaben, veröffentlicht. Auch die Hauptschwierigkeit, dass nur etwa die Hälfte der Korrespondenz erhalten ist, ein geschlossenes biographisches Bild gar nicht entstehen kann, wird in der »Einleitung « keineswegs verschwiegen. Nun ist aber bemerkenswert, dass versichert wird, der Band werde den wesentlichen Anforderungen trotzdem gerecht. In der Zusammenschau erhalte man dennoch einen » einigermaßen zutreffenden Eindruck « des behandelten Zeit-

I So Ernst Behler in »Der Stand der Friedrich Schlegel-Forschung «. In: Jabrbuch der Deutschen Schiller-Gesellschaft I (1957), S. 253-289, hier: S. 253. Ähnlich bereits Josef Körner, der gefordert hatte, die Forschung ruhen zu lassen, bis die editorischen Grundlagen erstellt seien. Friedrich Schlegel: Neue Philosophische Schriften. Hg. v. Josef Körner. Frankfurt a. M. 1935, S. 391. Zur Kritik des Denkmodells der »definitiven Editionen « vgl. Christian Benne/Ulrich Breuer: »Einleitung «. In: dies. (Hg.): Antike - Philologie - Romantik. Friedrich Schlegels altertumswissenschaftliche Manuskripte. Paderborn/München u. a. 20 I I, S. 7-I 4, hier: S. 8.

Friedrich Schlegel: Kritische Ausgabe seiner Werke. Bd. 25 
212 raums, »der Jahre 1799-1 802 in Jena, Berlin und Dresden « (S. LXXX). Der »Nachvollzug der geistigen Entwicklung « sei dennoch möglich, weil die »zentralen « Briefe an Fichte, Novalis, August Wilhelm, Schleiermacher und Tieck erhalten seien (ebd.).

Die Argumentation klingt optimistisch, die editorische Problematik scheint im Grunde vollständig kompensierbar zu sein. Dabei scheint die Auffassung zugrunde zu liegen, dass das biografische Briefkorpus über interne Strukturen der Konsistenz (wie Redundanz, Gewichtung) verfügt, die für semantische Stimmigkeit sorgen und sogar in der Lage sind, eine fragmentarische Überlieferung zu heilen. ${ }^{2}$ Aus geistesgeschichtlicher Sicht mag eine solche Annahme plausibel erscheinen. ${ }^{3}$ Angesichts methodischer Ansätze, deren Datenhunger das schmale Feld des literarischen Höhenkamms übersteigt (wie z. B. Konstellationsforschung ${ }^{4}$ und Sozialgeschichte), kann diese Sichtweise jedoch keineswegs als repräsentativ gelten.

Die editorische Problematik zeichnet sich noch schärfer ab, wenn man einbezieht, dass der Band, in dem es um »Höhepunkt und Zerfall der Romantischen Schule « geht, den Fokus vom Autor (hier: Friedrich Schlegel und Dorothea Veit) auf eine ganze Gruppe von Autoren ausweitet. Auch diesem Punkt versucht die Ausgabe gerecht zu werden. Auf die Gestaltung von Anmerkungen und Einleitung wird einzugehen sein.

Allerdings soll nicht übersehen werden, dass die Edition von Briefen der Romantischen Schule grundsätzliche Fragen aufwirft: Fragen zur Struktur der wissenschaftlichen Briefedition und den Möglichkeiten, Grenzen, die diese setzt, zu überschreiten. Im Rahmen der Werkausgabe basiert die Briefedition auf den Von- und An-Briefen des Autors. Diese

2 Eine ähnliche Argumentation begegnet bei Ernst Behler. Nach ihm geht es in der Abteilung »Briefe « der KFSA weniger um die Erschließung bisher unbekannter Texte als um Sammlung und Zusammenführung bekannter, aber bisher auf die verschiedensten Druckorte verstreuter Materialien. Der aus der Zusammenführung resultierende Mehrwert bestehe darin, dass erst bei dem »Ineinandergreifen der Briefe « und einer » fast automatischen Selbstkorrektur « derselben die Ereignisse im richtigen Licht erschienen. Ernst Behler: » Friedrich Schlegels erster Aufenthalt in Jena: Vom 6. August 1796 bis zum 3. Juli $1797 \ll$. In: Modern Language Notes 102 (1987), S. 545-569, hier: S. 545. - Dem Fegefeuer der Dekonstruktion war die Argumentation offenbar nie ausgesetzt.

3 Bekanntlich hat man in älteren Editionen sogar kürzende Eingriffe als zulässig betrachtet. Zur Kritik der Briefausgaben von Heinrich Finke und Martin Rottmanner vgl. KFSA 30 , S. XXVIII ff.

4 Zum Thema Konstellationsforschung vgl. neuerdings Dieter Henrich: Werke im Werden. Über die Genesis philosophischer Einsichten. München 20 I I (den Hinweis auf das Buch verdanke ich G. Naschert). 
>künstliche< Auswahl wird dem - im Falle der Romantischen Schule - 213

umfangreichen Netzwerk von Autoren, in dem Ideen und Inhalte realiter zirkulieren, nicht gerecht. So scheint es notwendig, Grenzen des Genres zu überschreiten; Editionen autorübergreifenden Zuschnitts werden relevant und Beispiele sind längst in Sicht. Zu erinnern ist in erster Linie an Josef Körners Krisenjahre der Frühromantik. Zu denken wäre ferner an die vorbildliche Ausgabe der Bonstettiana ${ }^{5}$, wo u. a. versucht wird, das Netzwerk, das eine gruppenbezogene Briefedition darstellt, durch entsprechende bildliche Mittel lesefreundlich zu präsentieren. Zu denken wäre vor allem auch an digitale Briefausgaben. Platzprobleme (selbst Josef Körners drei Bände umfassende Dokumentation kann nur eine Auswahl bieten) gibt es da kaum, umso mehr aber Möglichkeiten der Vernetzung von Dokumenten und Autoren. Vielleicht bietet die geplante Digitale Friedrich-Schlegel-Ausgabe einen weiteren Anlass zur Entwicklung eines Briefportals, das die Grenzen der an das Medium Buch gebundenen Editionen vergessen lässt.

Aber zurück zur Buchausgabe und dem vorliegenden Band. Die editorische Leistung ist anzuerkennen. Nur wissenschaftlich aufbereitete Texte werden weitgehend unverändert übernommen, zu den anderen werden teilweise neue Textfassungen erarbeitet. Bei der Herstellung des edierten Textes wird, wenn immer möglich, auf die Handschriften zurückgegriffen. Auf diese Weise gelingt es nicht selten, sinnentstellende Lesungen früherer Ausgaben zu korrigieren. Die Kommentierung ist kenntnisreich. In manchen Fällen werden überlieferte Datierungen von Briefen korrigiert. Das Kontextwissen, das die historischen Dokumente voraussetzen, wird präzise und gut dosiert zur Verfügung gestellt. In der Abteilung »Beilagen « wird stellenweise unveröffentlichtes Material erstmals publiziert. Gleichwohl ist partiell auch Kritik nötig. Sie betrifft in erster Linie die Verankerung des Bandes in Strukturen einer Kritischen Ausgabe, die, in die Jahre gekommen, sich heute als reformbedürftig erweist. So sind einige Bemerkungen zur Geschichte der Ausgabe vorauszuschicken.

I.

In dem 2008 zum 50. Geburtstag der Kritischen Friedrich-Schlegel-Ausgabe erschienenen Bericht von Ulrich Breuer, Till Dembeck und Maren

5 Bonstettiana. Historisch-Kritische Ausgabe der Briefkorrespondenzen Karl Viktor von Bonstettens und seines Kreises 1753-I832. Hg. v. Doris u. Peter Walser-Wilhelm u. a. Göttingen 2002. (Genaue bibliografische Daten unter www.bonstettiana.ch).

Friedrich Schlegel: Kritische Ausgabe seiner Werke. Bd. 25 
214 Jäger $^{6}$ wird beklagt, dass die Situation vor allem in der Abteilung »Briefe « kritisch sei, wo es nicht nur große Lücken gebe, sondern auch erhebliche Defizite hinsichtlich editorischer »Richtlinien und Standards « (S. I 69). Die KFSA ist in der Tat seit eh und je von den Entwicklungen der Editionstheorie relativ deutlich abgekoppelt. Man beruft sich auf die Praxis von Josef Körner und Hans Eichner. Im Übrigen gehört Theorie-Ferne historisch zu den Alleinstellungsmerkmalen $\operatorname{der} K F S A$ und insbesondere ihrer Abteilung »Briefe «. Die Defizite sind der Geschichte der Abteilung gleichsam eingeschrieben. Bezeichnend ist bereits, dass eine Abteilung Briefe in der ursprünglichen Planung der Ausgabe gar nicht vorgesehen war (KFSA 23, S. XXX).? Ohne editorische Fundierung werden in der ersten Hälfte der $1980 e r$ Jahre drei Briefbände publiziert. ${ }^{8}$ Erst 1987 erscheint - man beachte die Reihenfolge - der erste Band der Abteilung, von dem man editorische Grundsätze erwarten darf, der diese aber nicht enthält. ${ }^{9}$ Das Ende dieser Phase der Editionsgeschichte markiert ein kriti-

6 Ulrich Breuer/Till Dembeck/Maren Jäger: »Zum Stand der Kritischen Friedrich-Schlegel-Ausgabe «. In: Athenäum. Jahrbuch der Friedrich Schlegel-Gesellschaft I 8 (2008), S. 165-1 82.

7 Warum eine Abteilung Korrespondenz nicht vorgesehen war? Die ablehnende Haltung Josef Körners, der 1926 ein solches Unternehmen weder für möglich noch für nötig gehalten hatte, mag noch eine Rolle gespielt haben. Vgl. Die Brüder Schlegel. Briefe aus frühen und späten Tagen der deutschen Romantik. Bd. I: Briefe von und an Friedrich und Dorothea Schlegel. Hg. v. Josef Körner. Berlin I926, S. II.

8 In der auf ıo Bände geplanten dritten Abteilung der KFSA, welche die Korrespondenz von Friedrich und Dorothea Schlegel erschließen soll, erschienen 1980 ff. zunächst 3 Bände. 1980 erschienen die Bände 29 (Vom Wiener Kongre $\beta$ zum Frankfurter Bundestag, I8I4-1818. Hg. v. Jean Jacques Anstett) und 30 (Die Epoche der Zeitschrift Concordia, I818-1823. Hg. v. Eugène Susini). 1985 folgte Band 24 (Die Periode des Athenäum. Hg. v. Raymond Immerwahr). Da editorische Grundsätze für die Abteilung fehlen, beruft man sich, wenn überhaupt, auf eine Handvoll Regeln, » wie sie in dieser Ausgabe für den Druck von Handschriften aufgestellt wurden « (KFSA 29, S. XXVIII; KFSA 24, S. LII), die also nicht für die Edition der Briefe entwickelt sind, sondern aus der zweiten Abteilung (Schriften aus dem Nachlass) stammen und von dort übernommen werden.

9 Es handelt sich um KFSA 23 (Bis zur Begründung der romantischen Schule, 1788 1797. Hg. v. Ernst Behler). Das Theorie-Defizit zu beheben ist dieser erste Band der Abteilung »Briefe « allerdings auch nicht in der Lage. Dort erfährt man zunächst lediglich, dass eine Abteilung »Briefe « ursprünglich gar nicht geplant war. Der Grund, warum sie später trotzdem in die Planung Eingang fand, liege in der spezifischen Struktur von Schlegels Schriften aus dem Nachlass. Indem diese absichtlich » abgeschlossenen Werkcharakter « vermeiden, bilde die Korrespondenz eine »unlösbare Komponente von ihnen «. »Werk « und »Leben « seien bei Schlegel nicht trennbar, die Einheit seines Denkens sei »im wesentlichen eine 
scher Paukenschlag, die massive Kritik von Armin Erlinghagen, die I 987215 erschienen ist. ${ }^{10}$

Seit dieser fundamentalen Kritik befindet sich die KFSA in einer Situation des editorischen Umbruchs. Auf der einen Seite geht es um Behebung der Defizite, Kritik - die Konzeptvorgaben der KFSA müssen auf den Prüfstand -, Modernisierungsbedarf und das Bedürfnis der Teilhabe an den Neuerungen der (auch digitalen) Editionsphilologie. Auf der anderen Seite gibt es aber auch das Bedürfnis, bei all dem doch Kontinuität mit der bisher geleisteten Arbeit zu wahren und Konzepte, die den Bewährungstest bestehen, weiterzuführen.

Dieser Lage scheint sich der Herausgeber des neuen Briefbandes bewusst, indem er die Fähigkeit zum »Kompromiss « signalisiert, den Anspruch erhebt, der Kritik - » in Maßen « - zu folgen (S. LXXX) und gleichzeitig doch auch - zum Teil - an den »Prinzipien der KA« (S. LXXXI) festhalten will. - Wie das Vorhaben sich konkretisiert, soll nun verfolgt werden.

II.

Die bisher erschienenen Bände der Abteilung »Briefe « der KFSA sind mit ausführlicher Einleitung, Kommentierung und Namenregister versehen. Die Ausgestaltung entspricht im Wesentlichen Ernst Behlers Konzept der »erschließenden Philologie $\ll^{11}$, nach dem der Fokus auf dem Zusammenhang von Werk und Leben zu liegen hat.

biographische « (KFSA ${ }_{23}$, S. XXX). Was sich in solcher Rede zu erkennen gibt, ist Ernst Behlers Konzept der »erschließenden Philologie «, das, für Abteilung 2, die Nachlass-Schriften, entwickelt, nun auch als Grundlage für die Abteilung »Briefe « dienen soll. Spezifische Editionsgrundsätze für die Abteilung »Briefe « finden sich in $\operatorname{der} K F S A$ nicht.

I0 Armin Erlinghagen: »Wie kritisch ist die Kritische Friedrich-Schlegel-Ausgabe? Zur Fort- und Festschreibung fehlerhafter Entzifferungen im ersten Band der Dritten Abteilung «. In: Text. Kritische Beiträge 3 ( 1997), S. 85-120. Die Untersuchung am Text von KFSA 23 bringt gravierende Unzulänglichkeiten ans Licht, moniert nachdrücklich editorische Defizite und zeigt Wege auf, sie zu beheben.

I I Zu diesem Begriff vgl. Volker Deubel: »Die Friedrich Schlegel-Forschung «. In: Deutsche Vierteljahrsschrift für Literaturwissenschaft und Geistesgeschichte 47 (1973), S. 48-18 1, hier: S. 70 ff. Auf Sachregister, die Behler für die NachlassSchriften vorgesehen hatte (vgl. KFSA I I, »Vorbemerkung «) wird in der Abteilung $\gg$ Briefe « bisher verzichtet.

Friedrich Schlegel: Kritische Ausgabe seiner Werke. Bd. 25 
216 Der Ton einer biografischen Erzählung, den Ernst Behler in der Einleitung zum ersten Band der Abteilung vorgibt, ist auch im vorliegenden Band deutlich zu vernehmen. Das Schema Leben und Werk bestimmt den Aufbau. Allerdings nur weitgehend. So ist man nicht erstaunt, in der Einleitung, die insgesamt zwölf Abschnitte umfasst, Überschriften wie »Plato«, »Lyrik «, »Alarcos « und »Charakteristiken und Kritiken « zu begegnen, Kapiteln also, in denen Kontexte der genannten Werke oder Projekte behandelt werden. Aus dem Rahmen fällt dagegen die Darstellung von Promotion und Habilitation (zu der im Band neues Material vorgelegt wird), wo der Werkbezug (Schlegels Vorlesungen) hinter der prallen Geschichte eines »Skandals « zu verschwinden scheint. Dies gilt auch für die Kapitel über Friedrichs erotische Affären (seinen Beitrag zur »Jenaer Protobohème $\ll^{{ }^{12}}$ ), die seine Beziehung mit Dorothea begleiten, und eine wichtige (mitunter mathematische) Darstellung seiner Schuldenkrise ${ }^{13}$, Abschnitte also, die von vornherein eher rein biografische Kontexte reflektieren.

Wie man sieht, ist die editorische Vorgabe offenbar nicht punktgenau umzusetzen. Zu den Schwierigkeiten bei der Umsetzung des editorischen Konzepts, die sich hier zeigen, gesellen sich solche der Redundanz: Biographische Darstellungen gibt es auch in anderen Bänden der Ausgabe. Die einzelnen Darstellungen überlappen sich teilweise, woraus das Risiko der Verdoppelung und Wiederholung von Information resultiert. Auch hier wird also Modernisierungsbedarf erkennbar. ${ }^{14}$

I 2 Der Begriff findet sich in einer kürzlich erschienenen biografischen Skizze von Ulrich Breuer: »Friedrich Schlegel «. In: Wolfgang Bunzel (Hg.): Romantik. Epoche - Autoren - Werke. Darmstadt 2010, S. 60-75, hier: S. 68. Die Affären Friedrich Schlegels sind heute insofern Bildungswissen, als sie durch populäre Biografien zum weiblichen Personal der Romantik (Rahel von Varnhagen, Caroline Schelling, Sophie Mereau etc.) auch dem nicht-wissenschaftlichen Publikum vermittelt worden sind. Bei Oskar Walzel konnte man noch lesen: »Während Wilhelm von einem Weibe zum andern übergeht [...], ist Friedrich dem einmal gewählten Weibe treu geblieben.« (August Wilhelm und Friedrich Schlegel. In Auswahl. Hg. v. Oskar F. Walzel. Stuttgart I 892, S. XII).

I 3 Das Schuldenproblem wird letztendlich auf die prekäre Lage des freien Schriftstellers bezogen, der ohne »institutionellen Hintergrund « (S. LXXVIII) Ende des I 8. Jahrhunderts ökonomisch zum Scheitern verurteilt sei. Detaillierte Informationen über die chronische Schuldenkrise des Autors sind der eher auf Geisteskrisen ausgerichteten biografischen Studie von Harro Zimmermann nicht zu entnehmen. Harro Zimmermann: Friedrich Schlegel oder Die Sebnsucht nach Deutschland. Paderborn/München u. a. 2009.

I 4 Will man nach Alternativen zu Behlers Konzept Ausschau halten, wird man die Einleitungen der Briefbände der Kritischen Schleiermacher-Ausgabe nicht übergehen können. Anstelle von biografischer Erzählung liefern sie kalendarische Über- 
Neben der intellektuellen Biografie des Autors hat die Einleitung nach 217 Vorgabe der KFSA die Aufgabe, Blüte und Niedergang der Romantischen Schule darzustellen.

Relevant sind hier besonders Anfang und Ende der »Einleitung «, wo - einmal im Vorgriff, das andere Mal im Rückblick - eine Darstellung des besonderen Charakters der Romantischen Schule unternommen wird. Der Begriff wird nicht definiert. Neben der Bezeichnung »Schule «, die Schlegel selbst verwendet, werden Begriffe von Zeitgenossen wie $\gg$ Clique «, »Parthey« und »Kirche « genannt. In die Liste wären durchaus auch Begriffe wie »Orden « und »Gemeine «, die an anderer Stelle im Briefband begegnen, aufzunehmen. Später steuert der Herausgeber selbst weitere Begriffe (wie »Rezensionskartell« und »Briefgemeinschaft «) bei. Besondere Zustimmung findet aber ein zeitgenössisches Zeugnis, eine Interpretation der Romantischen »Schule « durch Schleiermacher. Diese ist in einem Brief von 1803 enthalten, dem ein längeres Zitat entnommen wird. Nach Schleiermacher handelte es sich bei dieser Schule um eine Gruppierung, deren Zusammenhalt weniger »offensiv « denn »defensiv « zu Stande kam, nicht durch gemeinsame Prinzipien sondern durch Druck von außen gewährleistet war: » man hat sie mit Gewalt aneinander gedrängt. « (S. LXXVII f.) Es versteht sich, dass diese Position mit Äußerungen anderer Zeitgenossen (Heinrich Steffen, A. W. Schlegel, Varnhagen von Ense etc.) und wissenschaftlicher Literatur zu vergleichen wäre.

$\mathrm{Zu}$ den Vorgaben der KFSA scheint es auch zu gehören, den in den einzelnen Bänden der Korrespondenz jeweils abgedeckten Zeitraum als in sich abgerundete lebensgeschichtliche Einheit, als biographische $\gg$ Epoche « $\left(K F S A_{23}, \mathrm{~S}\right.$. XXXI) oder wenigstens »Periode « $\left(K F S A{ }_{24}\right.$, S. XIX) erscheinen zu lassen. So ist zu fragen, wie die Periodisierung der Romantischen Schule im vorliegenden Band geschieht.

Als Zäsuren für Anfang und Ende der historischen Einheit dienen zwei Ortswechsel: der Umzug Friedrichs und Dorotheas von Berlin nach Jena im Herbst 1799 und die Reise, die I 802 zur Verlagerung des Wohnsitzes nach Paris führt. Nun lässt sich historischer Wandel nicht bruchlos mit Ortswechseln verrechnen. Schlegels Frühromantik, mit der wir es zu tun haben, beginnt nach überwiegendem Konsens bereits in der »Periode des Athenäums «, die im vorhergehenden Band (KFSA 24) behandelt wird. Um trotzdem eine Art Einheit für den behandelten Zeitraum zu

sichten, Abhandlungen zu speziellen Themen und analytische Beschreibung der Korrespondenz, wobei der Briefwechsel nach Personen geordnet und charakterisiert wird. Anzumerken wäre, dass die Kritische Schleiermacher-Ausgabe bei der Modernisierung der KFSA durchaus eine Rolle spielt. Darauf wird weiter unten eingegangen. 
218 retten, grenzt ihn die KFS A als »Höhepunkt « von der vorhergehenden »Periode « ab. In der Einleitung wird dieser Höhepunkt als relativ kurze Phase intensiver, produktiver und harmonischer Zusammenarbeit in der Jenaer Wohngemeinschaft Ende 1799 bis Anfang Mai I 800 gesehen, der endete, als August Wilhelm und Caroline Schlegel, denen sich später Schelling anschloss, Jena verließen. Wieder ist es ein Ortswechsel, der den Einschnitt markiert. Er gilt als »entscheidende persönliche Zäsur «, als »Augenblick « gar, von dem an es keine »Jenaer Frühromantik « mehr gab (S. LI). Was darauf folgt, sind zunehmende Zerwürfnisse und der allmähliche Rückgang gemeinschaftlicher Projekte. Weitere Ortswechsel erfolgen im Vorlauf der Reise nach Paris, die das Ende der Bewegung markiert und für den Autor einen »neuen Anfang « (S. LXXVII) mit sich bringt.

So bleibt die Darstellung der Romantischen Schule in der »Einleitung « auf skizzenhafte Umrisse beschränkt. Im Rahmen einer autorzentrierten Briefausgabe ist Literaturgeschichte aber wohl nicht anders zu meistern. Es handelt sich, wie bereits bemerkt, um eine Inkompatibilität von Gegenstand und editorischem Genre. Der Fall zeigt, dass die Kritik historischer Altlasten der KFSA auch die Vorgaben für Titel und Konzeption einzelner Bände einbeziehen muss.

Anzuschließen sind hier noch einige Bemerkungen zum Kommentar der Ausgabe. Obwohl es nach Josef Körner das Bestreben des Editors sein muss, » im Kommentar möglichst alles zu erklären « und die Erläuterungen zu einem »Archiv der Spezialforschung « auszubauen ${ }^{15}$, wird man selbst beim ausführlichsten Kommentar immer wieder auch auf Lücken stoßen. Hier einige Beispiele, die wichtige Gebiete romantischer Theorie betreffen.

Friedrichs Aktivitäten im Feld der Lyrik beschäftigen gleich zwei Kapitel der »Einleitung «. Das Machen von Gedichten hat Friedrich zu Anfang des Jahres I 800 in Jena erstmals probiert. Die »Stanzen Wuth « (S. 39) hat ihn gleich sehr in Bann gezogen. Ohne Hinweise auf Schlegels Theorie der Lyrik - er gilt als einer der » wichtigsten deutschsprachigen Theoretiker und Historiker der Lyrik um I $800 \ll^{16}-$ ist das kaum verständlich. Auch die Rede vom »Orden der Poesie « (S. 5 I) - sie dient einem der beiden Kapitel als Überschrift - hätte zur Dekodierung eine Anmerkung mit einschlägiger Theorie verdient. ${ }^{17}$ Theorie hat bei Schlegel

Is Briefe von und an August Wilhelm Schlegel. Zweiter Teil: Die Erläuterungen. Hg. v. Josef Körner. Zürich/Leipzig u. a. 1930, S. VI.

16 Dieter Burdorf: »Blätter, Rosen, Gärten. Zur Theorie des lyrischen Fragments beim jungen Schlegel «. In: Benne/Breuer (Hg.): Antike - Philologie - Romantik (s. Anm. I), S. I0 I-I 45, hier: S. Io4.

17 Charakteristische Aspekte von Schlegels Theorie der Lyrik behandelt bereits Dirk von Petersdorff: Mysterienrede. Zum Selbstverständnis romantischer Intellektueller. Tübingen 1996. 
nicht selten Vorlauf vor der Praxis, so dass letztere mitunter nur durch ers- 219 tere verständlich wird. Gerade auch auf die Ebene der Theorie sollte der Kommentar also verstärkt das Augenmerk richten. - Ein zweites Beispiel betrifft einen Konflikt Friedrichs mit Schleiermacher in Sachen Religion. Es geht um die Kommentierung von Briefen Friedrichs an Schleiermacher aus der Anfangsphase des Jenaer Aufenthalts. Die Anmerkungen (S. 369 ff.) machen wiederholt auf eine relativ hohe Dichte an Verschreibungen aufmerksam. Der Schreibduktus, erfährt man, sei Indikator der Entrüstung über Schleiermacher, mit dem es Streit in Sachen Religion gegeben hatte. Worin der Konflikt in der Sache genau beruhte, verraten die Anmerkungen aber nicht. Das Zerwürfnis hatte bereits Rudolf Haym untersucht. Auch in der Einleitung zu Band 24 der KFSA war es behandelt worden (S. XXVII). Dass Hinweise auf die Forschung in den Anmerkungen fehlen, kann man dem Kommentar allerdings nicht zum Vorwurf machen, da dieser » wissenschaftliche Sekundärliteratur « grundsätzlich nicht verzeichnen will (S. LXXXIV). Das ist bedauerlich, aber verständlich, wenn es sich um eine Maßnahme gegen ein weiteres Anschwellen des Buchumfangs handeln sollte. Allerdings sollte man dann wohl eher an Maßnahmen buchtechnischer Art denken. Eine Aufspaltung in Teilbände käme in Frage. Um die Lesefreundlichkeit zu erhöhen wäre an Möglichkeiten der Visualisierung von Information zu denken. Statistische Übersichten kämen in Betracht, ebenso wie tabellarische und graphische Darstellungen, ${ }^{18}$ die in anderen Editionen - z. B. in den Bänden der Bonstettiana ${ }^{19}$ - zum Einsatz gelangen.

Nach Einleitung und Kommentar soll nun die Aufmerksamkeit auf den Text gerichtet werden, den die Ausgabe bietet.

III.

Die »Einleitung « verweist auf die »Editionsprinzipien « der KFSA und den Versuch, diese durch $\gg$ Vergleich mit den neueren kritischen Ausgaben « zu Zeitgenossen Schlegels »im Sinne des Lesers zu verbessern «; genannt werden Fichte, Schelling, Schiller und Schleiermacher, wobei die »Anlehnung an die Schleiermacher-Edition « das entscheidende Moment darstellt (S. LXXXI).

I 8 Zum Konzept einer einer > graphisch< ausgerichteten Literaturgeschichte vgl. etwa Franco Moretti: Graphs, Maps, Trees. Abstract Models for Literary History. London/New York 2007.

i 9 Vl. etwa Band IX/I von Bonstettiana (s. Anm. 5).

Friedrich Schlegel: Kritische Ausgabe seiner Werke. Bd. 25 
220 Blickt man auf den Text, den der neue Band bietet, fallen sogleich zwei Neuerungen auf. Zunächst, dass er frei ist von spitzen Klammern, die in den früheren Bänden Einschübe bezeichnen. In der »Einleitung « heißt es dazu, dass in Abkehr von der bisherigen Praxis um einer »geschlosseneren Textform « willen auf » textkritische Zeichen im laufenden Text « global verzichtet werde. ${ }^{20}$ An denjenigen Stellen, an denen früher editorische Zeichen zu finden waren, sieht man nun nur noch hochgestellte Ziffern. Die Anmerkungen, auf die sie verweisen, finden sich im separaten Kommentar. - Was zweitens auffällt, ist die Häufigkeit der hochgestellten Ziffern im Text und der Anmerkungen im Apparat. Via Annotation werden nicht nur Einschübe, sondern auch Streichungen, Korrekturen, unsichere bzw. neue Lesungen, Emendationen etc. kenntlich gemacht. Durch Übernahme von Regeln der Schleiermacher-Ausgabe ist die »textkritische Dokumentation « verfeinert worden.

Ob diese Vermehrung der Noten aber wirklich im »Sinne des Lesers « ist? Der Lesefreundlichkeit dient auch die Mischung von textkritischen und erklärenden Anmerkungen keineswegs. Der meist als lästig empfundene Zwang zum Blättern zwischen Text- und Kommentarteil wird durch die Fülle der Noten unangenehm verstärkt. Lineare Lektüre, durch die Maxime der » geschlosseneren Textform « in Aussicht gestellt, wird durch das manuelle Exerzitium permanent unterlaufen. Dass dies auch die wissenschaftliche Nutzung erschwert, muss man nicht erläutern.

So meldet sich bereits nach kurzem Gebrauch Bedarf einer Reform der Reform. Auf den Prüfstand gehört die blockartige räumliche Trennung von Text und Apparat, wie diese in den Korrespondenz-Bänden der KFSA gehandhabt wird. Es ist dies eine Form der Darstellung, vor der - speziell im Hinblick auf die Edition von Briefwechseln - intensiv gewarnt wird. ${ }^{21}$ In der Schleiermacher-Edition, an der sich der vorliegende Band gerne ausrichtet, gibt es sie nicht. Dort findet man Text und Anmerkungen - textkritische und sonstige getrennt - jeweils auf der selben Seite. Warum dem Vorbild nicht auch in diesem Punkt gefolgt wurde, wird nicht erläutert.

20 Eckigen Klammern, die Auflösung von Abkürzungen und Einschübe des Editors kenntlich machen, begegnet man im Text aber nach wie vor. Leider fehlen Zeichen für Seitenwechsel, so dass der Brief als >Iconic Page $<$ gar nicht in den Blick genommen werden kann.

2 I »Wo soll man die Anmerkungen plazieren? Gewiß nicht ans Ende des Bandes «. Louis Le Guillou: »Prinzipien einer Briefwechsel-Edition «. In: Siegfried Scheibe (Hg.)/Christel Laufer (Red.): Zu Werk und Text. Beiträge zur Textologie. Berlin I991, S. 195-201, hier: S. 200. 
Problematisch scheint ferner eine weitere Trennung, welche in der 221 »Einleitung « propagiert wird: Es ist die Trennung von Archiv und Edition. Der Leser, an den sich die Briefausgabe richtet, heißt es in der »Einleitung «, sei ein literaturgeschichtlicher Leser, nicht der Schriftwissenschaftler. Damit wird begründet, dass innerhalb der Anmerkungen zu den einzelnen Briefen die Handschriftenbeschreibung eher kurz gehalten ist, Informationen über Wasserzeichen, den materiellen Überlieferungsträger, Umfang, Papier und Faltung etc. fehlen. Solche Informationen, die nur »Spezialisten für Schrifttypen, Schriftpsychologie, Papiersorten, Wasserzeichen « interessierten, müssten in den Archiven eingeholt werden (S. LXXXI). In anderen neueren Brief-Ausgaben muss man auf solche Informationen nicht verzichten. ${ }^{22}$ Sie sind ja gerade auch für den literarhistorischen Leser relevant, der, um zu verstehen, die Analyse des materiellen Trägers der Überlieferung einbeziehen muss. Der » bibliographic code «, heißt es z. B. in englisch-sprachiger Editionsforschung, sei grundsätzlich ebenso wichtig für das Textverstehen, wie der »linguistic code «, also der Inhalt der Botschaft, die in Texten vermittelt wird. ${ }^{23}$

Ein weiteres Thema, das sich im Hinblick auf den Text der Briefedition stellt, ist das der Authentizität ${ }^{24}$, der Art der Übereinstimmung, die zwischen editorischem Text und Original besteht. Der Begriff kann hier nicht diskutiert werden. Unstrittig dürfte sein, dass ein Faksimile eine andere Art von Authentizität besitzt als der Text einer Kritischen Ausgabe, der Streichungen, Korrekturen, Einfügungen etc. (alle Spuren der TextEntstehung, die das Faksimile weitgehend abbildet) zum großen Teil ausspart, um sie in Form textkritischer Anmerkungen in den Apparat zu deponieren. Hier ist von Interesse, dass in der Editionswissenschaft die Forderung gestellt wird, bei Brief-Editionen um der Authentizität willen mit diesem Verfahren zu brechen und den »Text von Briefen in möglichst originaler Gestalt zu edieren. « ${ }^{25}$ Dazu schlägt Hans Zeller ein neues Verfahren der Textdarstellung vor, das er als »integrale Darstellung « be-

22 Als neueres Beispiel sei der jüngst erschienene Band 6 II der historisch-kritischen Ausgabe der Goethe-Briefe genannt; Johann Wolfgang Goethe: Briefe. Bd. 6 II. Hg. v. Volker Giel unter Mitarb. v. Yvonne Pietsch u. Gerhard Müller. Berlin 2010.

23 Vgl. z. B.: George Bornstein (Hg.): The Iconic Page in Manuscript, Print, and Digital Culture. Ann Arbor 1998.

24 Vgl. Hans Zeller: »Authentizität in der Briefedition. Integrale Darstellung nichtsprachlicher Informationen des Originals «. In: editio I 6 (2002), S. 36-56. Ferner auch: Klaus Hurlebusch: »Divergenzen des Schreibens vom Lesen. Besonderheiten der Tagebuch- und Briefedition». In: editio 9 (1995), S. 18-36.

25 Zeller: »Authentizität in der Briefedition « (s. Anm. 24), S. 36.

Friedrich Schlegel: Kritische Ausgabe seiner Werke. Bd. 25 
222 zeichnet. Streichungen, Einschübe, Korrekturen etc. landen nicht etwa im Apparat, werden auch nicht - wie in typographischen Faksimiles, diplomatischen Transkriptionen - abgebildet, sie werden vielmehr im dargestellten Text via editorische Zeichen, Wechsel von Schriftarten, Schriftgraden etc. integriert. In der von Hans Zeller herausgegebenen historisch-kritischen Edition von C. F. Meyers Briefwechsel ist das Text-Modell realisiert. ${ }^{26}$ Für die KFSA-Briefedition zeichnet sich damit eine Alternative ab. Man wird sehen, ob es beim Streben $\gg$ nach einer geschlosseneren Textform « und strikter Trennung von Text und Apparat, Edition und Archiv, wie in KFSA 25 realisiert, bleiben wird.

IV.

Der neue Briefband erweist sich als eine Edition des Übergangs. Zur Disposition stehen die Prinzipien und Konzepte $\operatorname{der} K F S A$, die inzwischen in die Jahre gekommen sind. In manchen Fällen ist der neue Band innovativ. In anderer Hinsicht wird am Herkommen festgehalten. Gelegentlich zeigt die Edition die Offenheit einer Experimentierstation. ${ }^{27}$ Die Richtung, in die die Entwicklung gehen soll, heißt es gelegentlich, » muss sich noch herausstellen « (S. LXXXII). Die neu erscheinenden Briefbände werden sich damit auseinandersetzen müssen.

Dies gilt auch für die in Planung befindliche elektronische Version der KFSA. Einer der Vorteile des Mediums beruht in der Fähigkeit, verschiedene Text-Darstellungen nebeneinander realisieren zu können. ${ }^{28}$ Auch unterschiedliche Entwicklungen, die es in den noch ausstehenden Briefbänden geben könnte, wären also auf jeden Fall elektronisch aufgehoben. ${ }^{29}$

26 Vgl. v. a. Band drei der Ausgabe, der ausführliche Editorische Richtlinien enthält.

27 In den »Beilagen « kommen teilweise alternative Verfahren zur Anwendung. Beilage VII scheint als diplomatische Transkription intendiert. Beilage IV probiert ein anderes Verfahren der Annotation. In der »Einleitung « wird auf den experimentellen (»versuchsweisen «) Charakter (S. LXXXII) verwiesen.

28 Eine 2002 erstellte unter http://mut.mhn.de abrufbare Probe-Edition von Notizheften Friedrich Schlegels integriert edierten Text, Faksimiles der Handschrift und diplomatische Transkription.

29 Für Anregungen bei der Abfassung dieses Texts bin ich so mancher Diskussion mit interessierten Kollegen verpflichtet. Für konkrete Unterstützung danke ich Hermann Patsch, der mir seine Sammlung von Kopien der Autographen ausgeliehen hat, und Maren Jäger, die mir eine größere Anzahl von Digitalisaten zugänglich gemacht hat. 